\title{
The microtubule targeting agent PBOX-15 inhibits integrin-mediated cell adhesion and induces apoptosis in acute lymphoblastic leukaemia cells
}

\author{
JOANNE LYSAGHT ${ }^{1,2^{*}}$, NAVIN K. VERMA ${ }^{3 *}$, ELAINA N. MAGINN ${ }^{1}$, JACQUELINE M. RYAN $^{1}$, \\ GIUSEPPE CAMPIANI ${ }^{4}$, DANIELA M. ZISTERER ${ }^{5}$, D. CLIVE WILLIAMS ${ }^{5}$, \\ PAUL V. BROWNE ${ }^{1}$, MARK P. LAWLER ${ }^{1}$ and ANTHONY M. McELLIGOTT ${ }^{1}$
}

\begin{abstract}
${ }^{1}$ Department of Haematology, John Durkan Leukaemia Laboratories, Departments of ${ }^{2}$ Surgery and ${ }^{3}$ Clinical Medicine, Institute of Molecular Medicine, Trinity College Dublin, Dublin, Ireland; ${ }^{4}$ European Research Centre for Drug Discovery and Development, University of Siena, Italy; ${ }^{5}$ School of Biochemistry and Immunology, Trinity College Dublin, Dublin, Ireland
\end{abstract}

Received August 1,2012; Accepted September 25, 2012

DOI: 10.3892/ijo.2012.1688

\begin{abstract}
Although recent decades have seen an improved cure rate for newly diagnosed paediatric acute lymphoplastic leukaemia (ALL), the treatment options for adult ALL, T-cell ALL (T-ALL) and relapsed disease remain poor. We have developed a novel series of pyrrolo-1,5-benzoxazepine (PBOX) compounds and established their anticancer efficacy in a variety of human tumour cell types. Here, we demonstrate that PBOX-15 inhibits cell growth, and induces $\mathrm{G} 2 / \mathrm{M}$ cell cycle arrest and apoptosis in both T-ALL and B-cell ALL (B-ALL) cells. In addition, prior to PBOX-15-induced apoptosis, PBOX-15 decreases ALL cell adhesion, spreading and migration. Concurrently, PBOX-15 differentially down-regulates $\beta 1-$, $\beta 2$ - and $\alpha 4$-integrin expression in ALL cells and significantly decreases integrin-mediated cell attachment. PBOX-15 interferes with the lateral mobility and clustering of integrins in both B-ALL and T-ALL cells. These data suggest that PBOX-15 is not only effective in inducing apoptosis in ALL cells, but also has the potential to disrupt integrin-mediated adhesion of malignant lymphocytes, which represents a novel avenue for regulating leukaemic cell homing and migration.
\end{abstract}

\section{Introduction}

Acute lymphoblastic leukaemia (ALL) refers to a group of malignancies arising from lymphoid progenitors that may be of B- or T-cell lineage (B-ALL or T-ALL), resulting in prolifera-

Correspondence to: Dr Anthony M. McElligott, John Durkan Leukaemia Laboratories, Institute of Molecular Medicine, Trinity Centre for Health Sciences, St. James's Hospital, Dublin 8, Ireland

E-mail: tony.mcelligott@tcd.ie

${ }^{*}$ Contributed equally

Key words: acute lymphoblastic leukaemia, integrin, adhesion, apoptosis, microtubules tion and expansion of lymphoid blasts in bone marrow, blood and other organs. ALL has an overall incidence of 1-1.5 per 100,000 individuals and exhibits a bimodal age distribution with an early peak between ages 2-5 years (4-5 per 100,000 individuals, accounting for $80 \%$ of all childhood leukaemia), followed by a second peak after the age of 50 years (1). The treatment of ALL involves complex therapeutic programmes using intensive combination chemotherapy in dose- and timespecific sequences resulting in survival rates greater than $80 \%$ in children (2). However, there is a need for novel treatment options as only $30-40 \%$ of adults achieve long-term diseasefree survival and both childhood T-ALL and relapsed ALL are associated with poor clinical outcome $(3,4)$.

Microtubule targeting agents (MTAs) have been shown to be effective against ALL cells and vincristine is a key component of induction, consolidation and maintenance treatment protocols (3). We have previously described a novel MTA, pyrrolo-1,5-benzoxazepine-15 (PBOX-15), which exhibits anticancer activity against a variety of human tumour cell types, including those derived from both solid and haematological malignancies (5-10). Importantly, PBOX-15 and other related PBOX compounds display minimal toxicity against normal human blood and bone marrow cells, and are well tolerated by both tumour-bearing and healthy mice $(7,11)$.

MTA's interference with tubulin dynamics during mitotic spindle formation is well established; however, due to the critical role of microtubules in cell motility and homeostasis, the activity of MTAs may extend beyond inhibition of cytokinesis and subsequent induction of apoptosis. For example, we have shown that PBOX-15 induces apoptosis in chronic lymphocytic leukaemia (CLL) cells independent of cell cycle arrest and this effect has been described for other MTAs (7,12-14). In addition, MTAs have long been known to suppress immune function and have been used clinically in the treatment of other conditions besides cancer, including coronary restenosis and neointimal hyperplasia $(15,16)$.

A crucial role for integrins in the survival and homing of normal and neoplastic B- and T-cells cell is well-documented (17-19). Integrins trigger intracellular signalling pathways by 
forming macromolecular complexes with plasma membrane proteins and contribute to cell adhesion, mobility, homing and resistance to apoptosis $(18,20-22)$. Microtubules control integrin rearrangement and participate in the process of focal adhesions, which are sites of interactions between integrins and the cytoskeleton (23). We have previously shown that PBOX-15 inhibits integrin-induced migration of T-lymphocytes via disruption of tubulin dynamics prior to the induction of apoptosis (24). This suggested a potential anti-cancer activity for PBOX-15 involving disruption of leukaemic homing and infiltration, and subsequent induction of apoptosis. Here, we demonstrate anti-leukaemia potential of PBOX-15 by describing multiple effects of this agent in ALL cells: a pro-apoptotic activity, an inhibitory effect on cell adhesion and migration, down-regulation of integrin expression and disruption of integrin-microtubule interactions.

\section{Materials and methods}

Chemicals. PBOX-15 (pyrrolobenzoxazepine 4-acetoxy-5-(1naphthyl)naphtha pyrrolo[1,4]-oxazepine) was synthesised as previously described $(25,26)$ and prepared as a stock solution of $1 \mathrm{mM}$ in ethanol. Caspase inhibitors were obtained from Calbiochem (Darmstadt, Germany). Unless indicated, all other reagents and chemicals were obtained from Sigma-Aldrich Ireland Ltd. (Arklow, Ireland).

Cell culture. The T-ALL cell line CCRF-CEM and the B-ALL cell line SD-1 were obtained from the DSMZ cell bank (Braunschweig, Germany) for the purpose of this study. CCRF-CEM was established from the peripheral blood of a 3-year old girl with T-ALL at relapse (27) and SD-1 was established from the peripheral blood of an adult female with Philadelphia chromosome positive B-ALL (28). Both cell lines were cultured in complete medium [RPMI-1640 medium supplemented with $10 \%(\mathrm{v} / \mathrm{v})$ foetal bovine serum (FBS), $50 \mathrm{U} / \mathrm{ml}$ penicillin and $50 \mu \mathrm{g} / \mathrm{ml}$ streptomycin] under standard cell culture conditions.

Cell viability, cell cycle and apoptosis assays. Cell viability was determined by MTT assays (Cell Proliferation Kit I; Roche Applied Science, West Sussex, UK) according to the manufacturer's protocol. Cell cycle distribution was quantified by flow cytometric analysis of propidium iodide (PI; Invitrogen, UK) stained cells as described previously (10). In brief, following fixation and permeabilization with ethanol, cells were incubated with $25 \mu \mathrm{g} / \mathrm{ml}$ PI and $100 \mu \mathrm{g}$ RNase A (Gentra Systems Inc., USA) before analysis using a FACSCalibur and CellQuest software (BD Biosciences, Oxford, UK). Doublets were excluded and approximately 30,000 events were acquired for each sample. Apoptosis was detected by co-staining of cells with fluorescein isothiocyanate (FITC)-conjugated Annexin V (IQ Products, The Netherlands) and PI (29), and quantified using a FACSCalibur and CellQuest software. In some experiments caspase inhibitors were added $1 \mathrm{~h}$ prior to PBOX-15 treatment.

Real-time impedance sensing. Real-time monitoring of adhesion and spreading of cells was performed using xCELLigence system as per manufacturer's instructions (Roche Applied Science). This system measures electrical impedance (which depends on cell number, degree of adhesion, spreading and proliferation of the cells) across interdigitated micro-electrodes integrated on the bottom of 96-well E-plates. CCRF-CEM cells were seeded at a density of $1 \times 10^{4}$ cells/well in $100 \mu 1$ media and allowed to attach onto the electrode surface over time. The electrical impedance was automatically recorded every $15 \mathrm{~min}$ and expressed as an arbitrary unit called the Cell Index. Cells were treated with PBOX-15 either prior to seeding or at $21 \mathrm{~h}$ post-seeding when the cells had strongly adhered to the surface of the E-plate.

Cell adhesion assay. Adhesion of cells on anti-integrin antibody or fibronectin coated surfaces was detected and subsequently quantified using a cell based high content analysis (HCA) system. The assay operates on the principle of fully automated fluorescent microscopy described previously (30-32) and allows multiplexing of key reporter parameters including cell-adhesion. Untreated or PBOX-15 pre-treated cells were added to fibronectin, anti- $\alpha 4-, \beta 1-$, or $\beta 2$-integrin antibody coated 24-well or 96-well plates (Nunc, Roskilide, Denmark) at a density of $2 \times 10^{5}$ or $1 \times 10^{4}$ cells/well respectively. Cells were allowed to adhere $2 \mathrm{~h}$ after which the non-adherent cells were removed by gentle washing three times in phosphate-buffered saline (PBS). Following fixation with 3\% paraformaldehyde, adherent cells were fluorescently stained with Rhodaminephlloidin and Hoechst to visualize cell morphology and the nuclei, respectively. Plates were scanned using IN Cell Analyzer 1000 automated microscope (GE Healthcare, Buckinghamshire, UK) and images were acquired in a stereology configuration of nine randomly selected fields per well using a 10x objective. The quantification of cell adherence was subsequently performed using IN Cell Investigator software.

Transwell migration assay. The transwell migration assay was performed using standard procedure. Briefly, untreated or PBOX-15 pre-treated cells $\left(5 \times 10^{5}\right.$ in $100 \mu \mathrm{l}$ serum-free medium) were loaded in the upper chamber of a 24-well Costar ${ }^{\circledR}$ Transwell $^{\mathrm{TM}}$ inserts (Costar, Cambridge, MA) with a pore size of $5 \mu \mathrm{m}$. Filters then were transferred to wells containing $600 \mu \mathrm{l}$ medium with or without $20 \%$ FBS. Cells were allowed to migrate for $2 \mathrm{~h}$ and transmigrated cells were quantified by automated microscopy using an IN Cell Analyzer 1000. All assays were performed in triplicate.

Integrin expression analysis by flow cytometry. Single cell suspensions were added to individual polystyrene tubes and Fc receptors were blocked by incubating with FBS. Cells were washed and incubated for $30 \mathrm{~min}$ at $4^{\circ} \mathrm{C}$ with anti-human $\alpha 4-\mathrm{PE}, \beta 1-\mathrm{FITC}$, or $\beta 2$-FITC integrin antibodies or appropriate isotype control antibodies (BD Pharmingen, CA, USA). Flow cytometry was performed using a FACSCalibur with CellQuest software. PI staining was used to exclude dead cells from the analysis. Twenty thousand events were collected for each sample, gated to exclude debris and illustrated as histograms.

Confocal microscopy. Immunofluorescent staining and confocal microscopy was performed as described previously (30). Briefly, cells were seeded $\left(2 \times 10^{4}\right.$ cells/well) in a Lab-Tek 8-well Permanox ${ }^{\circledR}$ slide (Nalge Nunc, NY, USA). After treatments, as indicated in particular experiments, cells were fixed with $3 \%$ paraformaldehyde, immunostained with anti- $\alpha 4-, \beta 1-$, 
CCRF-CEM $24 \mathrm{~h}$

A

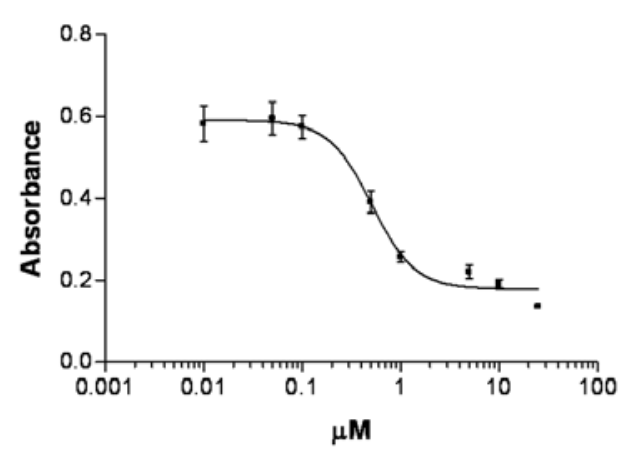

B
CCRF-CEM

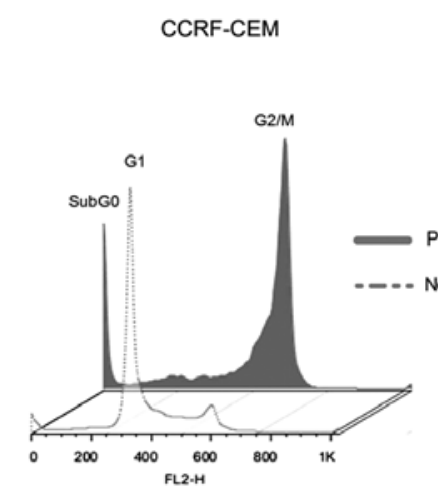

D

SD-1

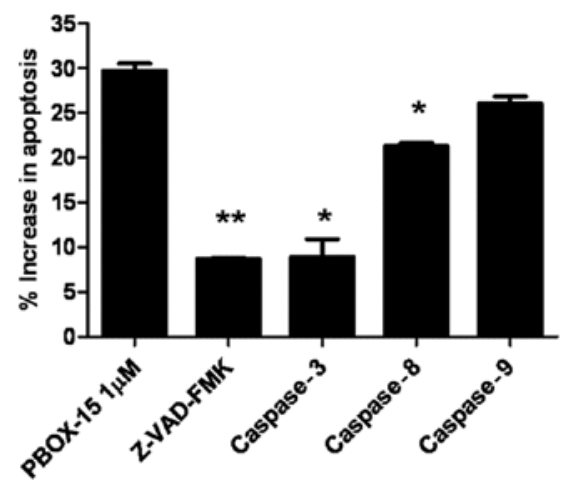

SD-1 24h

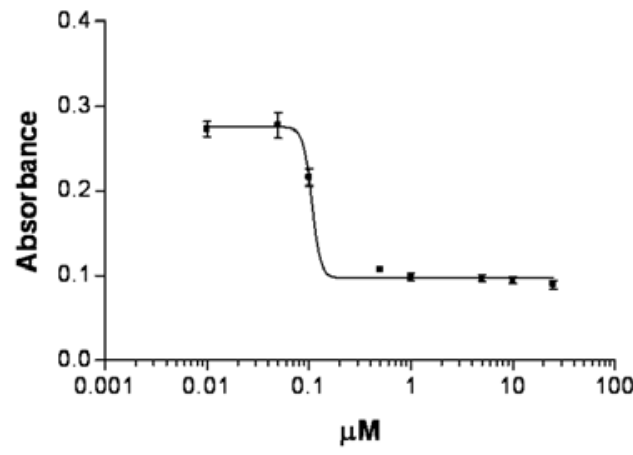

C

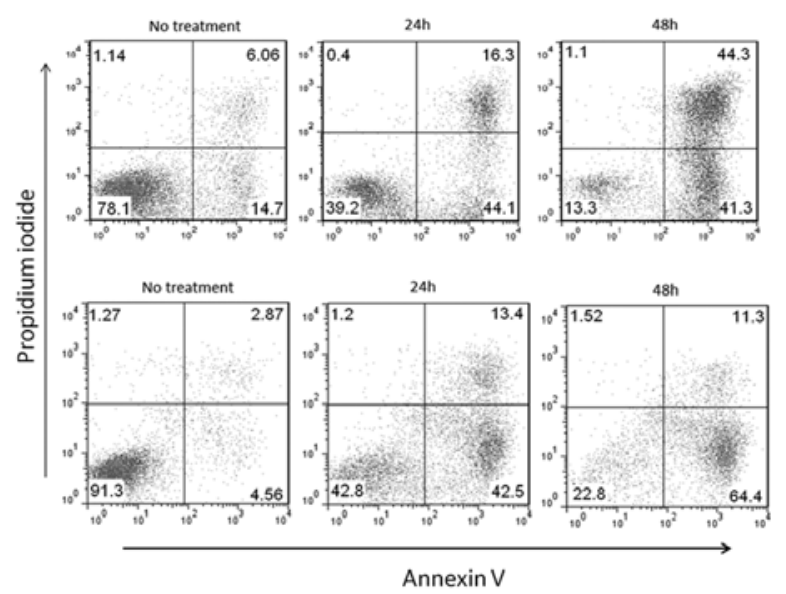

SD-1

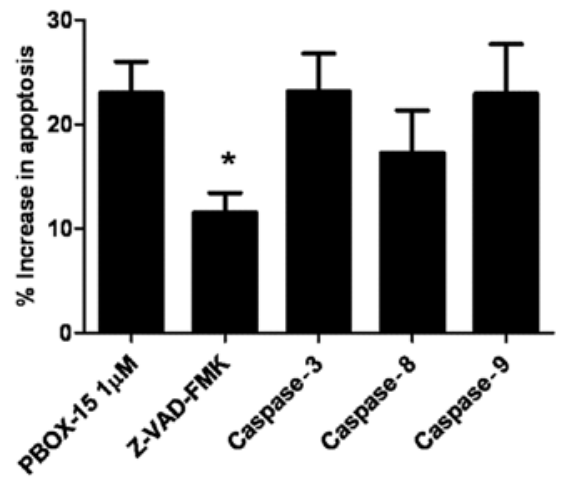

Figure 1. PBOX-15 inhibits cell growth, induces G2/M cell cycle arrest and apoptosis. A, Cell viability was assessed by MTT assay following treatment of both CCRF-CEM and SD-1 cells with PBOX-15, for 24 h. Bars: SEM of triplicate samples. B, CCRF-CEM and SD-1 cells underwent cell cycle arrest in the G2/Mphase following 24-h treatment with $1 \mu \mathrm{M}$ PBOX-15. Following treatment cells were stained with PI and DNA content (FL2-H) was analyzed by flow cytometry. $\mathrm{C}$, Apoptosis induction was assessed in CCRF-CEM cells (top panels) and SD-1 cells (bottom panels) following no treatment (ethanol vehicle control) or $1 \mu \mathrm{M}$ PBOX treatment at 24 or $48 \mathrm{~h}$ by flow cytometry analysis of FITC-Annexin V/PI staining. Numbers represent the percentage of total cells in each quadrant. D, Inhibition of PBOX-15 induced apoptosis was assessed using pharmacologic inhibitors of caspases: pan-caspase inhibitor (z-VAD-fmk, $100 \mu \mathrm{M})$; caspase-3 inhibitor [Z-D(OMe)QMD(OMe)-FMK, $50 \mu \mathrm{M}$ ]; caspase-8 inhibitor [Z-IE(OMe)TD(OMe)-FMK, $50 \mu \mathrm{M}$ ]; caspase-9 inhibitor (Ac-LEHD-CMK, $50 \mu \mathrm{M})$. Cells were treated directly with $1 \mu \mathrm{M}$ PBOX-15 or pre-treated for $1 \mathrm{~h}$ with specific caspase inhibitors before addition of PBOX-15. After $24 \mathrm{~h}$, apoptosis was assessed by Annexin V/PI assay. ${ }^{*} \mathrm{p}<0.01,{ }^{* *} \mathrm{p}<0.001, \mathrm{n}=3$, by Student's t-test compared with PBOX-15 treated cells. Column data are illustrated as mean increase in apoptosis compared to ethanol vehicle control treated cells \pm SEM. All data shown are representative of 3 independent experiments.

or $\beta 2$-integrin antibody (eBiosciences, Hatfield, UK) for $1 \mathrm{~h}$, and then permeabilized in $0.1 \%$ Triton $\mathrm{X}-100$. The slides were then blocked by the addition of $3 \%(\mathrm{v} / \mathrm{v})$ goat serum in PBS, counterstained with FITC- or TRITC-conjugated anti- $\alpha$-tubulin antibody. Images were aquired using a $63 \mathrm{x}$ oil emersion objective on a Zeiss 510 LSM confocal microscope system (Carl Zeiss Jena GmbH, Jena, Germany). At least 20 different microscopic fields were analyzed for each sample.

\section{Results}

PBOX-15 inhibits cell growth, induces G2/M cell cycle arrest and apoptosis in both $T$ - and B-ALL cells. To investigate if PBOX-15 could affect ALL cell growth and viability, we performed a MTT-based cell proliferation and toxicity assay. PBOX-15 caused a dose-dependent reduction in viability of CCRF-CEM and SD-1 cells after $24 \mathrm{~h}$ with $\mathrm{IC}_{50}$ values of less 
A

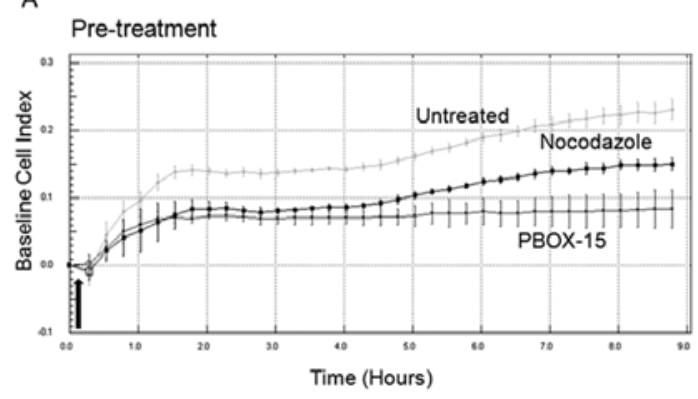

PBOX-15 treatment at $21 \mathrm{~h}$

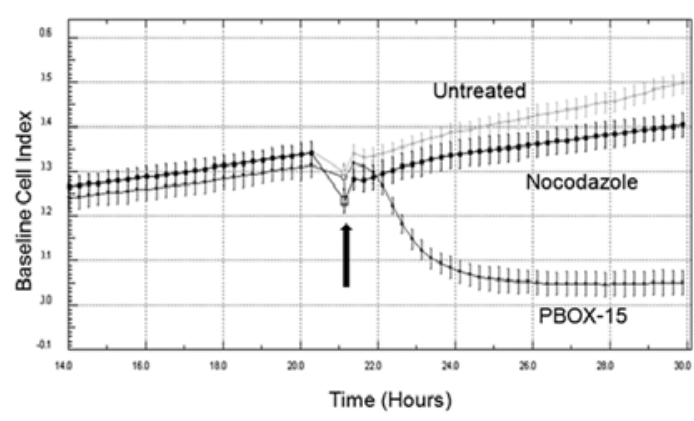

B

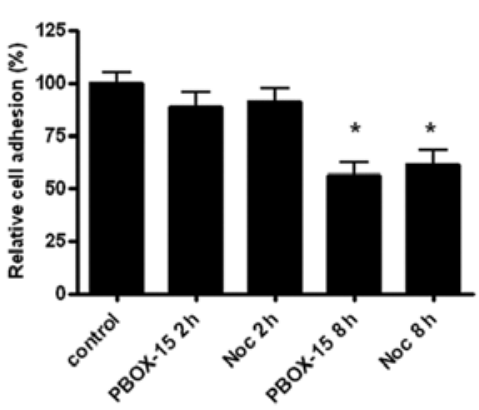

C

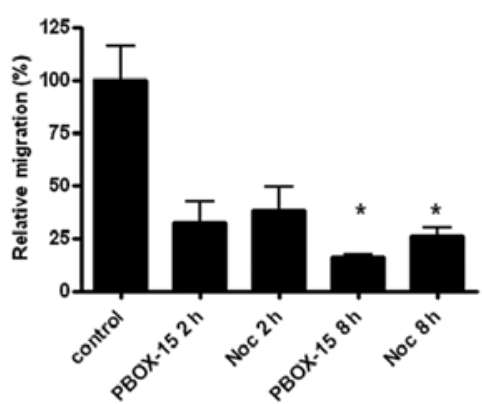

SD-1

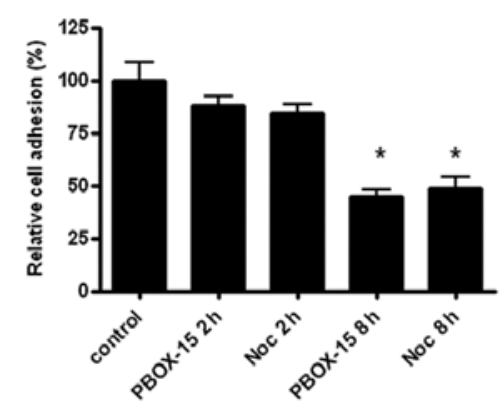

SD-1

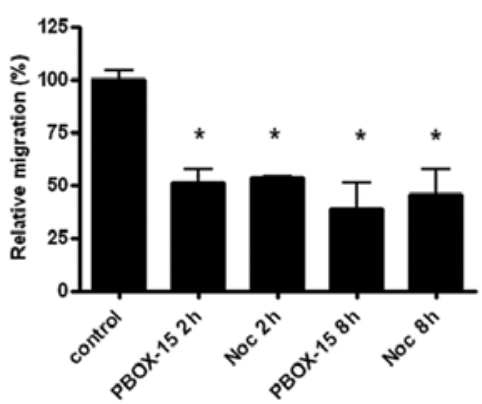

Figure 2. PBOX-15 inhibits adhesion and migration of T-ALL cells. A, Real-time monitoring of CCRF-CEM cell adhesion and spreading following $1 \mu \mathrm{M}$ PBOX-15 and $1 \mu \mathrm{M}$ nocodozole treatment was performed using xCELLigence electronic sensing system. This system measures electrical impedance across interdigitated micro-electrodes integrated on the bottom of 96 -well tissue culture plates by micro-electronic sensor technology. Cells $\left(1 \times 10^{4}\right)$ were seeded in the E-plate and allowed to attach onto the electrode surface over time. The electrical impedance of untreated (ethanol vehicle control) cells and cells treated at time $0 \mathrm{~h}$ (arrow) with $1 \mu \mathrm{M}$ PBOX-15 or $1 \mu \mathrm{M}$ nocodozole was recorded every $15 \mathrm{~min}$ (top panel). Untreated (ethanol vehicle control) cells were allowed to adhere and spread for $21 \mathrm{~h}$ after which time (arrow) one group was treated with $1 \mu \mathrm{M}$ PBOX-15 or nocodozole and monitored for additional $9 \mathrm{~h}$ (bottom panel). The cell impedance, expressed as an arbitrary unit (Cell Index), was automatically calculated on the xCELLigence system. Data are illustrated as means \pm SEM. Data shown are representative of 3 independent experiments. B, CCRF-CEM and SD-1 cells were treated with $1 \mu \mathrm{M}$ PBOX-15 or $1 \mu \mathrm{M}$ nocodozole as indicated and allowed to adhere to fibronectin coated plates for 2 or $8 \mathrm{~h}$. Plates were scanned using an IN Cell Analyzer 1000 automated microscope and cell adherence was quantified using IN Cell Investigator software. Column data are illustrated as means \pm SEM. ${ }^{*} \mathrm{p}<0.01, \mathrm{n}=3$, by Student's t-test compared to control cells. C, Following treatment with $1 \mu \mathrm{M}$ PBOX-15 or $1 \mu \mathrm{M}$ nocodozole for 2 and $8 \mathrm{~h}$, migration potential of CCRF-CEM and SD-1 cells were assessed by a transwell migration assay. Significant inhibition of ALL cell migration compared to ethanol vehicle control was observed as indicated. Column data are illustrated as means \pm SEM. ${ }^{*}<0.05$, $\mathrm{n}=3$, Student's t-test.

than $1 \mu \mathrm{M}$ (Fig. 1A). In order to investigate the mechanism of this PBOX-15-induced effect on ALL cells, we performed cell cycle analysis. A G2/M-phase cell cycle arrest was observed following 24-h treatment with $1 \mu \mathrm{M}$ PBOX-15 in both T- and B-ALL cells. A notable increase in the subG0 peak was evidenced in both cell lines, indicative of increased numbers of apoptotic cells (Fig. 1B). We have previously shown this concentration and duration of exposure to PBOX-15 to be minimally toxic to normal CD19+ B lymphocytes (7). In order to confirm and quantify PBOX-15-induced apoptosis, CCRF-CEM and SD-1 cells were treated with $1 \mu \mathrm{M}$ PBOX-15 for 24 or $48 \mathrm{~h}$, stained with Annexin V and PI, and then analyzed by flow cytometry. A substantial increase in both early apoptotic (lower right quadrants) and late apoptotic (upper right quadrants) cells was detected at both 24 and $48 \mathrm{~h}$ post-treatment for both cell lines (Fig. 1C). To investigate the role of caspases in mediating PBOX-15-induced apoptosis in ALL cells, specific pharmacologic inhibitors of caspase activity were used. Pre-treatment of cells with the pan-caspase inhibitor, z-VAD-fmk, significantly inhibited PBOX-15-induced apoptosis in CCRF-CEM cells $(\mathrm{p}<0.001)$ and SD-1 cells $(\mathrm{p}<0.01)$, suggesting a requirement for caspase activity. Pre-treatment with specific caspase- 3 and 8 inhibitors significantly reduced PBOX-15-induced apoptosis in T-ALL cells only $(\mathrm{p}<0.01)$ (Fig. 1D).

PBOX-15 treatment alters the adhesion and migration of ALL cells. We next investigated the effect PBOX-15 on ALL cell adhesion, spreading and migration prior to induction of cell cycle arrest and subsequent apoptosis. CCRF-CEM cells grow both in suspension and as an adherent monolayer in culture, allowing for dynamic analysis of cell adhesion and cell spreading using the xCELLigence electronic sensing system. CCRF-CEM cells were treated with either $1 \mu \mathrm{M}$ PBOX-15 or the well-characterized microtubule targeting agent nocodozole and cellular adhesion and spreading of the cells were monitored in real-time every $15 \mathrm{~min}$ for up to $8 \mathrm{~h}$. Control cells with normal cell adherence showed continuously increasing Cell Index values, displaying a upwardly trending curve, as the cells adhered and spread. Treatment of cells with PBOX-15 or nocodozole inhibited their ability to adhere to the plate surfaces and spread as observed by the curve plateaus, with $\mathrm{PBOX}-15$ treatment resulting in a more pronounced effect (Fig. 2A, top panel). In order to determine if PBOX-15 could exert its effect on pre-adhered CCRF-CEM cells, cells were allowed to adhere for $21 \mathrm{~h}$ prior to treatment 
A CCRF-CEM

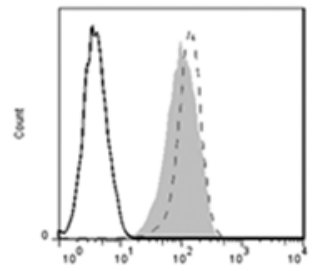

$\beta 1$ integrin

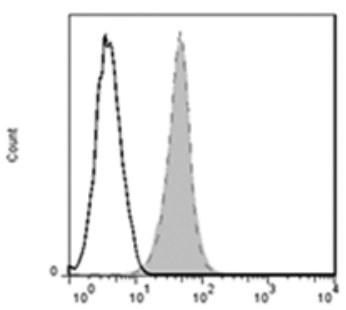

$\beta 2$ integrin

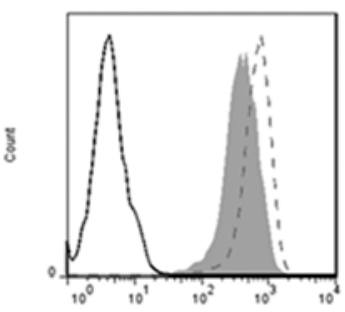

$\propto 4$ integrin
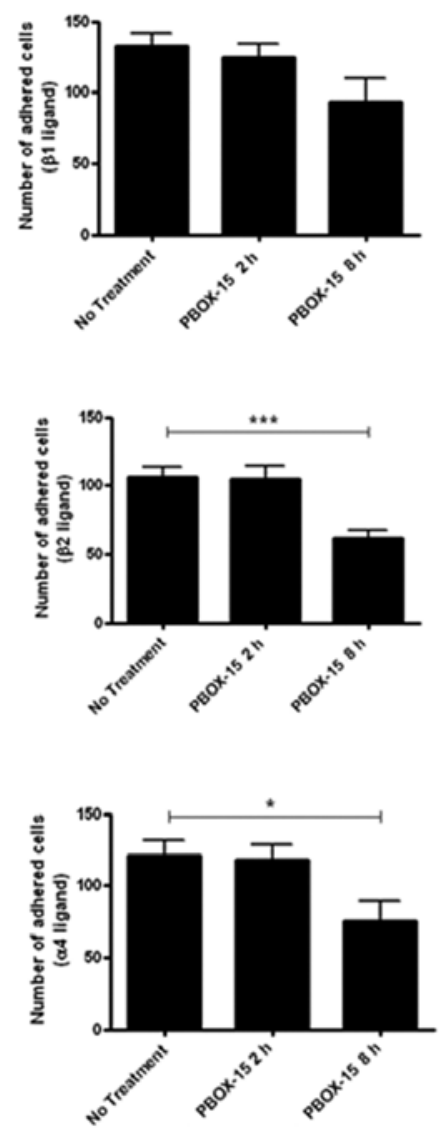

B SD-1

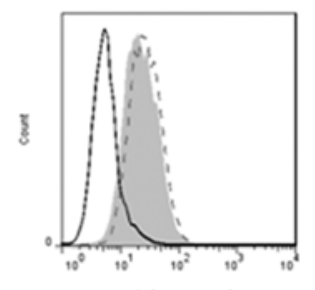

$\beta 1$ integrin

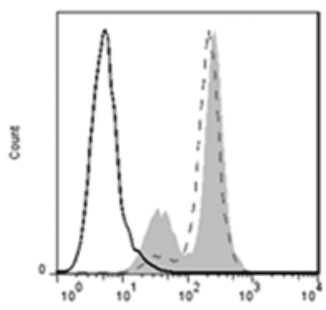

$\beta 2$ integrin

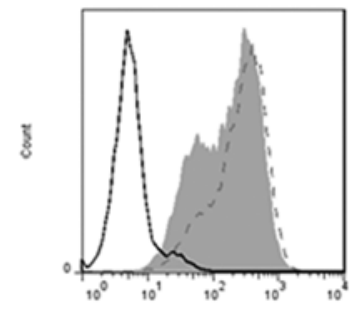

$\propto 4$ integrin
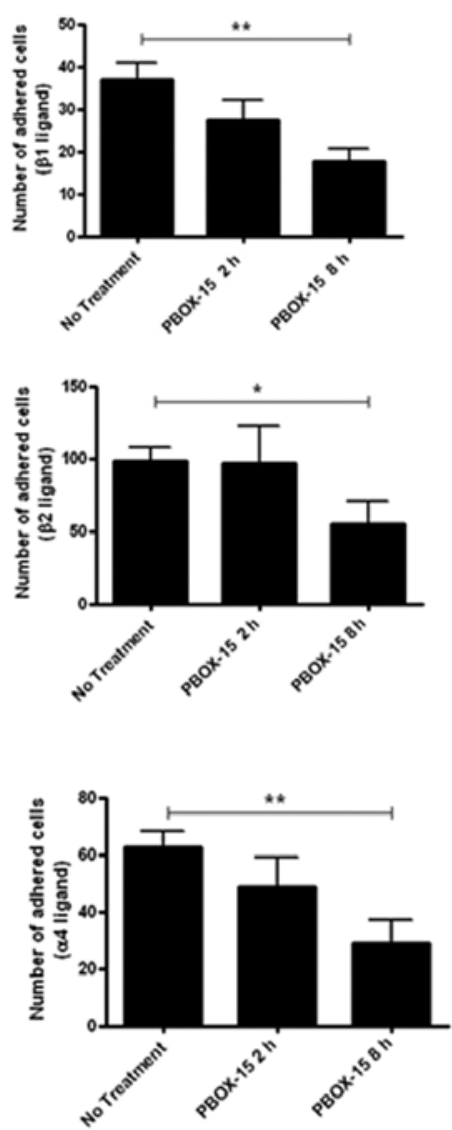

Figure 3. PBOX-15 significantly alters integrin-mediated adherence of ALL cells. A, CCRF-CEM and B, SD-1 cells were treated with $1 \mu \mathrm{M}$ PBOX-15 for 8 h, stained for $\alpha 4$-PE, $\beta 1$-FITC or $\beta 2$-FITC integrins, and analyzed by flow cytometry. Histograms show isotype control ( - ), untreated (ethanol vehicle control) cells (---), PBOX-15 treated cells (grey area). Adhesion of cells on anti- $\alpha 4, \beta 1$, or $\beta 2$-integrin antibody coated surfaces was subsequently quantified using a cell-based high content analysis system. Adherent cells were fluorescently stained with Rhodamine-phalloidin and Hoechst to visualize cell morphology and the nuclei respectively. Images were acquired using an automated microscope IN Cell Analyzer 1000 and quantified by IN Cell Investigator software. Column data are illustrated as means \pm SEM. ${ }^{*} \mathrm{p}<0.05,{ }^{* *} \mathrm{p}<0.01,{ }^{* * *} \mathrm{p}<0.001, \mathrm{n}=3$, by Student's t-test compared to 'no treatment' (vehicle control)

(Fig. 2A, bottom panel). Following PBOX-15 treatment, cellular adherence was rapidly and substantially decreased as evidenced by a decline in cellular electrical impedance within $30 \mathrm{~min}$. Interestingly, we observed a much more pronounced effect on pre-adhered cells treated with PBOX-15 compared to cells treated with nocodozole.

To further investigate these early PBOX-15-induced phenotypic changes in ALL cells, we examined the effects of PBOX-15 on ALL cell adhesion to the extracellular matrix protein fibronectin and on cell migration. Pre-treatment of both CCRF-CEM and SD-1 cells for $8 \mathrm{~h}$ with PBOX-15 significantly reduced cell adhesion to fibronectin, with nodcodozole having a similar effect ( $<<0.01$; Fig. 2B). Both PBOX-15 and nocodozole potently inhibited ALL cell migration in a transwell migration assay after 2-h exposure, with significant inhibition of both CCRF-CEM and SD-1 cell migration after 8 -h treatment ( $<<0.05$; Fig. 2C). To ensure that the effects of PBOX-15 on cellular adherence, morphology and migration were not due to the induction of cell death, PBOX-15 treated CCRF-CEM and SD- 1 cells $(1 \mu \mathrm{M}$ for 2 or $8 \mathrm{~h}$ ) were stained with FITC-Annexin V and PI, and apoptosis was assessed by flow cytometry. No increase in apoptotic cells was observed at up to 8-h treatment compared with untreated cells (data not shown).
PBOX-15 down-regulates integrin expression and decreases integrin-mediated adhesion in ALL cells. In order to determine if the decreased ALL cell adhesion and migration due to PBOX-15 treatment was associated with dysregulation of integrins involved in adhesion to fibronectin and lymphocyte trafficking, the expression levels of $\alpha 4$ (CD49d), $\beta 1$ (CD29) and $\beta 2$ (CD18) integrins were assessed by flow cytometry following treatment with $1 \mu \mathrm{M}$ PBOX-15 for $8 \mathrm{~h}$. A decrease in $\alpha 4$ - and $\beta 1$-integrin expression was observed in the CCRF-CEM cells (Fig. 3A), while a substantial decrease in $\alpha 4$ - and $\beta 2$-integrin was observed in SD-1 cells (Fig. 3B). To investigate if this had a functional impact on integrin-mediated ALL cell attachment, plates were coated with anti- $\alpha 4-$, anti- $\beta 1$ - or anti- $\beta 2$-integrin and adhesion of control or PBOX-15-treated cells ( 2 or $8 \mathrm{~h}$ ) was assessed using an HCA system. Following $8 \mathrm{~h}$ of PBOX-15 treatment, a significant reduction of CCRF-CEM cell adhesion on $\beta 2(\mathrm{p}<0.001)$ and $\alpha 4(\mathrm{p}<0.05)$ integrins was observed (Fig. 3A). Similarly in the SD-1 cells, a significant decrease in adhesion via $\beta 1(\mathrm{p}<0.01), \beta 2(\mathrm{p}<0.05)$ and $\alpha 4(\mathrm{p}<0.01)$ integrins was observed (Fig. 3B).

PBOX-15 interferes with lateral mobility and clustering of integrins in ALL cells. An intact and dynamic microtubule 
A. CCRF-CEM
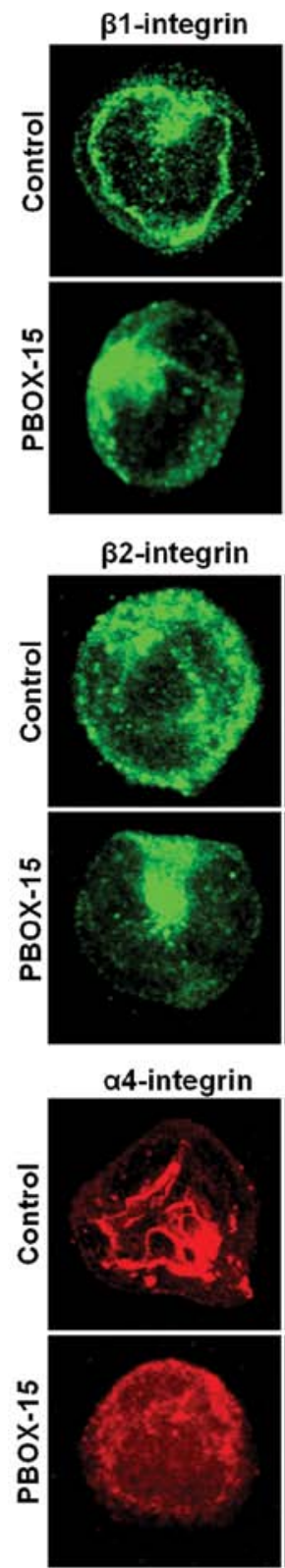

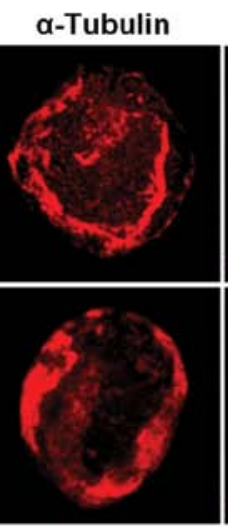

a-Tubulin

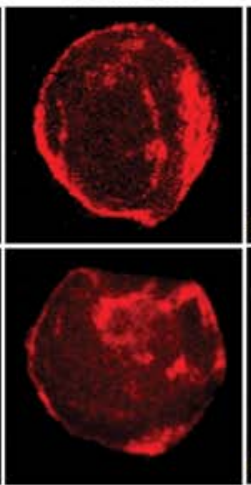

a-Tubulin

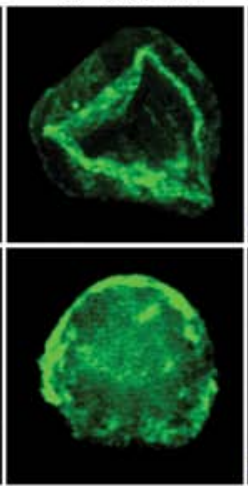

Merge

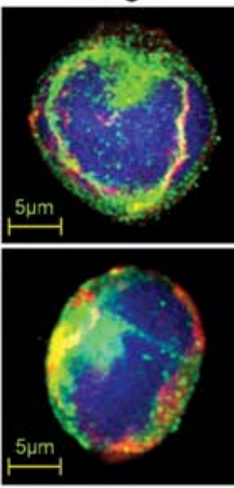

Merge

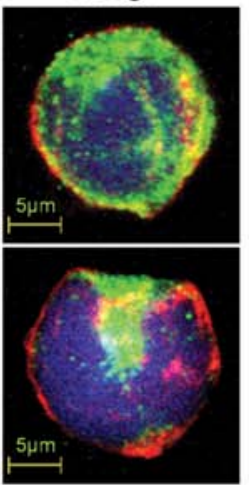

Merge

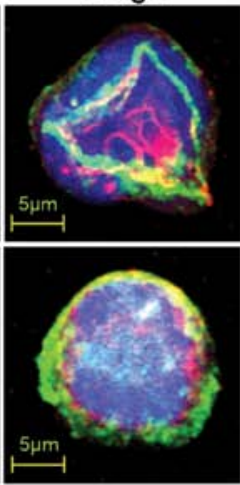

B. SD-1
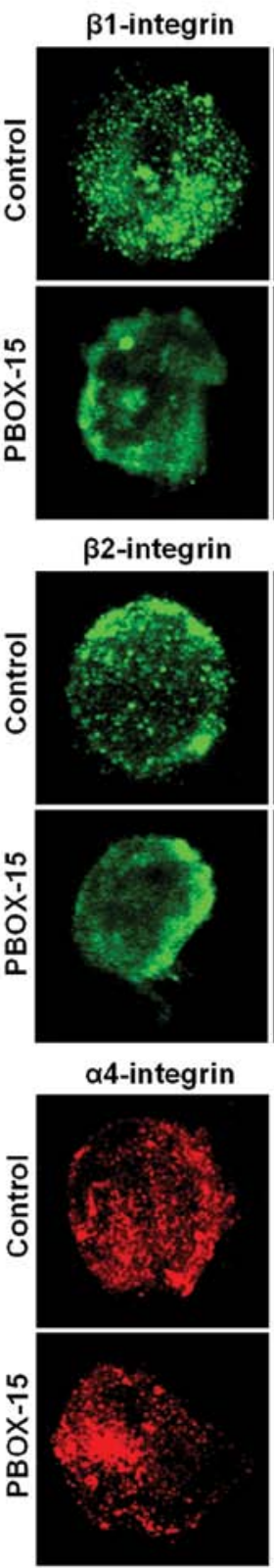

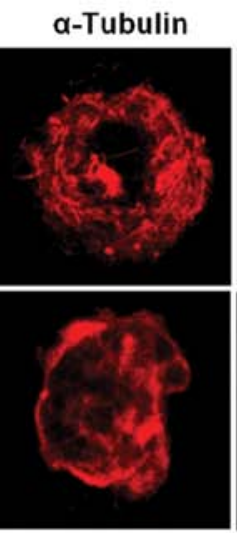

a-Tubulin

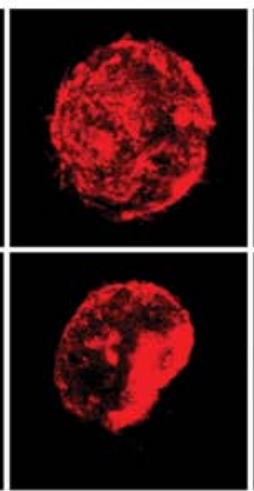

$\alpha-T u b u l i n$
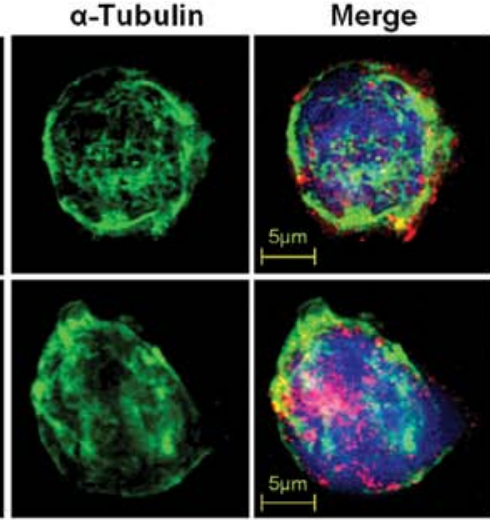

Figure 4. PBOX-15 treatment alters the lateral mobility and clusturing of integrins in both T- and B-ALL cells. A, CCRF-CEM and B, SD-1 cells were untreated (ethanol vehicle control) or treated with $1 \mu \mathrm{M}$ PBOX-15 and immunostained with anti- $\alpha 4$-PE, $\beta 1$-FITC, or $\beta 2$-FITC integrin and then counterstained with FITCor TRITC-conjugated anti- $\alpha$-tubulin and Hoechst. Fluorescence microscopy was performed using a $63 \mathrm{x}$ oil emersion objective on a Zeiss 510 LSM confocal microscope system. At least 20 different microscopic fields were analyzed for each sample.

system is required for the cytoskeletal constraint on the lateral mobility of integrins, and, thus, integrin-mediated adhesion. Moveover, the microtubule cytoskeleton is known to participate in the control of integrin avidity (23). To further investigate if the PBOX-15-induced decrease in adhesion of ALL cells to $\alpha 4$-, $\beta 1$ - or $\beta 2$-integrin ligands was due to alteration in lateral mobility and clustering of integrins, ALL cells were treated with $1 \mu \mathrm{M}$ PBOX-15 for $8 \mathrm{~h}$ and, subsequently, were immunostained for $\alpha 4-, \beta 1-$, or $\beta 2$-integrins and $\alpha$-tubulin. Confocal microscopy revealed that the disruption of the cell membrane distribution and clustering of integrins was concurrent with the disruption of microtubule architecture in both CCRF-CEM (Fig. 4A) and SD-1 cells (Fig. 4B).

\section{Discussion}

The treatment outcome for ALL has improved dramatically in recent decades with a cure rate exceeding $80 \%$ in paediatric disease. However, a number of challenges remain which necessitate improved therapeutic options: up to $25 \%$ of patients relapse, resulting in relapsed ALL being the leading cause of cancer related death in children and young adults; adult ALL continues to have a poor prognosis; and T-ALL is associated with unfavourable clinical features such as high white blood cell counts, bulky adenopathy and involvement of the central nervous system with resulting poor clinical outcomes. Here, we show anti-cancer activity of a novel MTA 
compound, PBOX-15, in cell line models representing poor prognostic subgroups of ALL: relapsed childhood T-ALL (CCRF-CEM) and BCR-ABL positive adult B-ALL (SD-1).

Similar to our previous studies on the effect of PBOX-15 on other cancer cell types $(5,6,10)$, we showed that PBOX-15induced $\mathrm{G} 2 / \mathrm{M}$ cell cycle arrest and growth inhibition was associated with the induction of apoptosis in B-ALL and T-ALL cells. A pan-caspase pharmacological inhibitor inhibited PBOX-15-induced apoptosis in both T-ALL and B-ALL cells, while caspase- 3 and 8 pharmacological inhibitors inhibited apoptosis in T-ALL cells. We have recently shown caspase-8-dependent PBOX-15-induced apoptosis in CLL and myeloma cells using a pharmacological inhibitor and siRNA knockdown of caspase-8 expression $(7,10)$. However, caspase inhibitors only partially inhibited PBOX-15-induced apoptosis suggesting that caspase-independent mechanisms are also involved in PBOX-15-induced apoptosis in ALL cells (33).

Our previous study showing that $\mathrm{PBOX}-15$ rapidly inhibits integrin-induced T-cell migration (24) allowed us to postulate that PBOX-15 may potentially inhibit cell homing and mobility, and hence, the metastatic potential of ALL cells. In addition, gene expression studies on PBOX-15-treated CCRF-CEM and SD-1 cells revealed alteration of the expression of genes involved in multiple cellular pathways, including resistance to apoptosis and chemotaxis, suggesting that PBOX-15 may affect multiple aspects of the ALL cell phenotype (unpublished data). In this study, we used an impedanciometry technique to obtain qualitative information in real-time on the biological status of CCRF-CEM cells, including degree of adhesion, spreading and proliferation. PBOX-15 reduced the Cell Index of CCRF-CEM cells compared to control cells within $1 \mathrm{~h}$, indicating that this effect on the phenotype of the cells was independent of cell cycle arrest and subsequent apoptosis, and suggesting that PBOX-15 rapidly inhibited the adhesion and spreading of the cells $(34,35)$. Of note is the more pronounced and sustained effect of PBOX-15 on the cells compared to treatment with nocodazole (Fig. 2A). This may indicate that PBOX-15 has a distinct mechanism of action compared to other tubulin depolymerising agents and, indeed, we have shown differences in the mechanism of action of PBOX-15 compared to nocodozole in CLL cells (7). The effect of PBOX-15 was more pronounced on pre-adhered cells suggesting that PBOX-15 may have a more potent effect on cell spreading compared to nocodazole. However, as both PBOX-15 and nocodazole had similar effects on cell adhesion to fibronectin and cell migration, the significance of the $\mathrm{xCel}-$ ligence data requires further investigation.

The PBOX-15-induced inhibition of ALL cell adhesion to fibronectin and cell migration further suggests that PBOX-15 may affect ALL homing and trafficking. Lymphocyte trafficking to secondary organs involves tissue and lymphocyte specific multistep adhesion cascades. Integrins are among the most dynamic adhesion molecules and are critically important for cell migration. Activated integrins, particularly members the $\alpha 4, \beta 1$ and $\beta 2$ subfamilies, mediate the firm adhesion of circulating lymphocytes to vascular cells and are crucial for lymphocyte trafficking (36). Very late antigen-4 (VLA-4), an $\alpha 4-, \beta 1$-integrin dimer, is a receptor for fibronectin and has been associated with B-cell survival and activation of the phosphatidylinositol-3-kinase signalling pathway in acute leukaemia (17,37). Recently, VLA-4 expression has been identified as a poor prognostic marker and potential therapeutic target in relapsed childhood B-ALL (38). We observed significantly reduced $\alpha 4-, \beta 1$ - and $\beta 2$-integrin mediated adhesion in PBOX-15-treated B-ALL cells concurrently with down-regulation of the respective integrin subunits. In comparison, down-regulation of the integrin subunits in PBOX-15-treated T-ALL cells was less pronounced, although $\alpha 4-$ and $\beta 2$-integrin mediated adhesion was significantly reduced. Importantly, we show that the cell surface distribution and clustering of $\alpha 4-, \beta 1-$ and $\beta 2$-integrins is disrupted in both ALL cell lines following PBOX-15 treatment. A crucial role of the microtubule cytoskeleton in the control of integrin avidity has been established (23). The cytoskeleton is intimately involved in the dynamic regulation of the adhesive state of integrins, switching from sufficiently strong binding to maintain cell-cell contacts, to a state that allows cells to dissociate and migrate. The microtubule system is not only important for driving this membrane remodelling (via either an increase or decrease of integrin avidity) but also acts as a platform to bring together surface receptors and recruit activable enzymes and substrates. Integrins interact with the microtubule cytoskeleton through association of their cytoplasmic domains and the MTA colchicine, nocodazole and taxol have been shown to interefere with integrin clustering (23). Our data on the altered distribution and clustering of integrins, as a result of PBOX-15-mediated microtubule disruption, may suggest a role for integrin avidity in signalling mechanisms and/or gene regulation in ALL cells.

Overall, this study indentifies PBOX-15 as an agent which targets multiple aspects of the ALL cell phenotype and, as such, warrants further investigation of its potential as a novel therapeutic agent for the treatment of ALL.

\section{Acknowledgements}

This study was funded by the Children's Leukaemia Research Project and the Irish Cancer Society. N.K.V. and equipment were part-funded by Health Research Board (HRB) and Higher Education Authority (HEA) of Ireland.

\section{References}

1. Jemal A, Tiwari RC, Murray T, et al: Cancer statistics, 2004. CA Cancer J Clin 54: 8-29, 2004.

2. Pui CH, Robison LL and Look AT: Acute lymphoblastic leukaemia. Lancet 371: 1030-1043, 2008.

3. Pui $\mathrm{CH}$ and Evans WE: Drug therapy - treatment of acute lymphoblastic leukemia. N Engl J Med 354: 166-178, 2006.

4. Faderl S, O'Brien S, Pui CH, et al: Adult acute lymphoblastic leukemia concepts and strategies. Cancer 116: 1165-1176, 2010.

5. Mulligan JM, Greene LM, Cloonan S, et al: Identification of tubulin as the molecular target of proapoptotic pyrrolo-1,5-benzoxazepines. Mol Pharmacol 70: 60-70, 2006.

6. Greene LM, Campiani G, Lawler M, Williams DC and Zisterer DM: BubR1 is required for a sustained mitotic spindle checkpoint arrest in human cancer cells treated with tubulintargeting pyrrolo-1,5-benzoxazepines. Mol Pharmacol 73: 419-430, 2008.

7. McElligott AM, Maginn EN, Greene LM, et al: The novel tubulintargeting agent pyrrolo-1,5-benzoxazepine-15 induces apoptosis in poor prognostic subgroups of chronic lymphocytic leukemia. Cancer Res 69: 8366-8375, 2009. 
8. Nathwani SM, Butler S, Fayne D, et al: Novel microtubuletargeting agents, pyrrolo-1,5-benzoxazepines, induce apoptosis in multi-drug-resistant cancer cells. Cancer Chemother Pharmacol 66: 585-596, 2010

9. Forde JC, Maginn EN, McNamara G, et al: Microtubule-targetingcompound PBOX-15 radiosensitizes cancer cells in vitro. Cancer Biol Ther 11: 421-428, 2011.

10. Maginn EN, Browne PV, Hayden P, et al: PBOX-15, a novel microtubule targeting agent, induces apoptosis, upregulates death receptors, and potentiates TRAIL-mediated apoptosis in multiple myeloma cells. Br J Cancer 104: 281-289, 2011.

11. Bright SA, McElligott AM, O'Connell JW, et al: Novel pyrrolo1,5-benzoxazepine compounds display significant activity against resistant chronic myeloid leukaemia cells in vitro, in ex vivo patient samples and in vivo. Br J Cancer 102: 1474-1482, 2010.

12. Vilpo JA, Koski T and Vilpo LM: Selective toxicity of vincristine against chronic lymphocytic leukemia cells in vitro. Eur $\mathrm{J}$ Haematol 65: 370-378, 2000

13. Beswick RW, Ambrose HE and Wagner SD: Nocodazole, a microtubule depolymerising agent, induces apoptosis of chronic lymphocytic leukaemia cells associated with changes in Bcl-2 phosphorylation and expression. Leuk Res 30: 427-436, 2006.

14. Moon EY and Lerner A: Benzylamide sulindac analogues induce changes in cell shape, loss of microtubules and G(2)-M arrest in a chronic lymphocytic leukemia (CLL) cell line and apoptosis in primary CLL cells. Cancer Res 62: 5711-5719, 2002.

15. Aisenberg AC and Wilkes B: Studies on suppression of immune responses by periwinkle alkaloids vincristine + vinblastine. J Clin Invest 43: 2394-2403, 1964

16. Park SJ, Shim WH, Ho DS, et al: A paclitaxel-eluting stent for the prevention of coronary restenosis. N Engl J Med 348: 1537-1545, 2003.

17. Manabe A,Murti KG, Coustansmith E, et al: Adhesion-dependent survival of normal and leukemic human-B lymphoblasts on bone-marrow stromal cells. Blood 83: 758-766, 1994.

18. De la Fuente MT, Casanova B, Moyano JV, et al: Engagement of alpha 4 beta 1 integrin by fibronectin induces in vitro resistance of B chronic lymphocytic leukemia cells to fludarabine. J Leukoc Biol 71: 495-502, 2002

19. Scimone ML, Alfantis I, Apostolou I, von Boehmer H and von Andrian UH: A multistep adhesion cascade for lymphoid progenitor cell homing to the thymus. Proc Natl Acad Sci USA 103: 7006-7011, 2006

20. Cox D, Brennan M and Moran N: Integrins as therapeutic targets: lessons and opportunities. Nat Rev Drug Discov 9: 804-820, 2010.

21. Astier AL, Svoboda M, Hinds E, De Beaumont R, Munoz O and Freedman AS: Integrins regulate survival of pre-B-ALL cells through differential IAP and caspase-7 ubiquitination and degradation. Leukemia 18: 873-875, 2004.

22. Streuli CH and Akhtar N: Signal co-operation between integrins and other receptor systems. Biochem J 418: 491-506, 2009.

23. Zhou XM, Li JX and Kucik DF: The microtubule cytoskeleton participates in control of beta(2) integrin avidity. J Biol Chem 276 : 44762-44769, 2001

24. Verma NK, Dempsey E, Conroy J, et al: A new microtubuletargeting compound PBOX-15 inhibits T-cell migration via post-translational modifications of tubulin. J Mol Med 86: 457-469, 2008 .
25. Campiani G, Nacci V, Fiorini I, et al: Synthesis, biological activity, and SARs of pyrrolobenzoxazepine derivatives, a new class of specific 'peripheral-type' benzodiazepine receptor ligands. J Med Chem 39: 3435-3450, 1996

26. McGee MM, Gemma S, Butini S, et al: Pyrrolo[1,5]benzoxa(thia) zepines as a new class of potent apoptotic agents. Biological studies and identification of an intracellular location of their drug target. J Med Chem 48: 4367-4377, 2005.

27. Foley GE, Lazarus H, Farber S, Uzman BG, Boone BA and McCarthy RE: Continuous culture of human lymphoblasts from peripheral blood of a child with acute leukemia. Cancer 18 522-529, 1965.

28. Dhut S, Gibbons B, Chaplin T and Young BD: Establishment of a lymphoblastoid cell-line, Sd-1, expressing the p190 bcr-abl chimeric protein. Leukemia 5: 49-55, 1991.

29. Vermes I, Haanen C, Steffens-Nakken H and Reutelingsperger C: A novel assay for apoptosis. Flow cytometric detection of phosphatidylserine expression on early apoptotic cells using fluorescein labelled Annexin V. J Immunol Methods 184: 39-51, 1995.

30. Verma NK, Dourlat J, Davies AM, et al: STAT3-stathmin interactions control microtubule dynamics in migrating T-cells. J Biol Chem 284: 12349-12362, 2009.

31. Mohamed BM, Verma NK, Davies AM, et al: Citrullination of proteins: a common post-translational modification pathway induced by different nanoparticles in vitro and in vivo. Nanomedicine 7 : $1181-1195,2012$

32. Mohamed BM, Verma NK, Prina-Mello A, et al: Activation of stress-related signalling pathway in human cells upon $\mathrm{SiO} 2$ nanoparticles exposure as an early indicator of cytotoxicity. J Nanobiotechnology 9: 29, 2011.

33. McGrath LB, Onnis V, Campiani G, Williams DC, Zisterer DM and McGee MM: Caspase-activated DNase (CAD)-independent oligonucleosomal DNA fragmentation in chronic myeloid leukaemia cells; a requirement for serine protease and $\mathrm{Mn}^{2+}$ dependent acidic endonuclease activity. Apoptosis 11: 1473-1487, 2006.

34. Whipple RA, Matrone MA, Cho EH, et al: Epithelial-tomesenchymal transition promotes tubulin detyrosination and microtentacles that enhance endothelial engagement. Cancer Res 70: 8127-8137, 2010.

35. Tiruppathi C, Malik AB, Del Vecchio PJ, Keese CR and Giaever I: Electrical method for detection of endothelial cell shape change in real time: assessment of endothelial barrier function. Proc Natl Acad Sci USA 89: 7919-7923, 1992.

36. Von Andrian UH and Mackay CR: Advances in immunology: T-cell function and migration - two sides of the same coin. N Engl J Med 343: 1020-1033, 2000.

37. Matsunaga T, Takemoto N, Sato T, et al: Interaction between leukemic-cell VLA-4 and stromal fibronectin is a decisive factor for minimal residual disease of acute myelogenous leukemia. Nat Med 9: 1158-1165, 2003.

38. Shalapour S, Hof J, Kirschner-Schwabe R, et al: High VLA-4 expression is associated with adverse outcome and distinct gene expression changes in childhood B-cell precursor acute lymphoblastic leukemia at first relapse. Haematologica 96: 1627-1635, 2011. 